\title{
LA METAPHORISATION DE L'EXISTENCE LIQUIDE : CARNETS DE NAUFRAGE DE GUILLAUME VIGNEAULT
}

\author{
Veronika ČERNÍKOVÁ \\ Université de Bohême du Sud à České Budějovice
}

\begin{abstract}
En): In 2000, Zygmunt Bauman published an essay called Liquid Modernity in which contemporary society is compared to liquids: unstable and insecure, obsessed with satisfying desires, incapable of long-term relationships. In the same year, Carnets de naufrage, the first novel by the young Quebec writer Guillaume Vigneault is published, which presents an identical perception of society and, moreover, uses the liquid metaphor.

The article not only introduces the Bauman's concept of liquid society and connects it to the Vigneault's vision, but mainly focuses on how the Quebec author creates and shapes the liquid metaphor.
\end{abstract}

Keywords (En): contemporary Quebec novel; Vigneault; Bauman; liquid modernity; liquid society; liquid metaphor

Mots-clés (Fr) : roman québécois contemporain ; Vigneault ; Bauman ; modernité liquide ; société liquide ; métaphore liquide

Fils d'un célèbre chanteur québécois, Guillaume Vigneault est un véritable enfant de son siècle. Né en 1970, il sort à trente ans son premier roman, Carnets de naufrage $(2000)^{1}$, qui pourrait être sous-titré «Confessions de la génération liquide », la génération hantée par le vide et la solitude, la précarité et l'incertitude. Son début littéraire est un livre matériellement liquide dont les pages suintent des métaphores mouillées. Il est vrai que Guillaume Vigneault n'est pas le premier à s'emparer de la métaphore de l'eau pour exprimer le vide vécu par l'homme postmoderne. Rappelons deux exemples frappants : La salle de bain que JeanPhilippe Toussaint publie en 1985 et Extension du domaine de la lutte publiée par Michel Houellebecq en 1994.

Une grande partie de La salle de bain se déroule dans les locaux éponymes où le narrateur coule des heures agréables, tandis que sa partenaire trouve quelque chose de desséchant dans son refus de les quitter. Le choix de cet ancrage spatial non seulement mouille l'imaginaire et les champs lexicaux, mais surtout aborde symboliquement la question de la fuite de temps, l'une des préoccupations principales de la société postmoderne (BAUMAN, 2000 : 91-129).

Le narrateur d'Extension du domaine de la lutte s'adresse à son homologue, le lecteur modèle, homme postmoderne en proie à l'absolue solitude et la sensation de l'universelle vacuité. Il l'invite de façon suggestive à plonger avec lui dans l'eau froide et amère et à se souvenir du moment où il a quitté le domaine de la règle pour entrer dans le domaine de la lutte. Loin du bord, l'homme continue à nager même s'il ne croit plus à l'existence d'une autre rive et que sa vie ressemble de plus en plus à une noyade.

1 Toutes les références aux Carnets de naufrage, ainsi que les citations, proviennent de l'édition Vigneault Guillaume (2001), Carnets de naufrage, Montréal, Boréal. 
Vers la fin du millénaire, la métaphore liquide noie aussi le discours théorique de Zygmunt Bauman qui scrute les phénomènes sociaux actuels. Son concept de la modernité liquide veut remplacer celui de la postmodernité (JUIGNET, 2017). En effet, BAUMAN distingue deux phases de la modernité : «solid modernity » et «liquid modernity». La modernité solide s'applique bien à ce que Houellebecq classifie comme le domaine de la règle où existent des formes sociales stables et bien définies et où l'immobilité concerne les masses aussi bien que les élites. Par contre le domaine de la lutte houellebecquien et la postmodernité lyotardienne correspondent à la phase liquide de la modernité où la liberté est payée par l'incertitude, le changement, la précarité et l'insécurité (JUIGNET, 2017) et où les élites se déplacent sans le moindre problème tandis que pour les masses immobilisées, c'est le monde entier qui est en mouvement (BAUMAN, $2000: 58$ ). Le héros de Vigneault ressemble comme une goutte d'eau à l'individu liquéfié de Bauman. Sciemment ou pas, l'auteur québécois matérialise, littéralement et littérairement, son concept de société liquide.

\section{La société liquide selon Zygmunt Bauman}

Dans son essai Liquid Modernity, Zygmunt BAUMAN explique que contrairement aux corps solides, les liquides ne peuvent pas conserver leur forme quand ils sont exposés à une force extérieure : ils subissent des changements constants (2000: 1). C'est pourquoi la fluidité et la liquidité représentent les métaphores qui saisissent le mieux la nature de notre ère d'instantanéité (« era of instantaneity ») aux liens humains fragiles et aux engagements précaires (BAUMAN, 2000: 14-15). Bauman détermine cinq points par lesquels se manifeste la liquéfaction de la société : l'émancipation, l'individualité, l'accélération, le travail et la communauté.

L'émancipation, qui libère l'homme de la société tout en le responsabilisant, entraîne l'agonie de l'indécision et la peur de l'échec. Bien qu'il dispose d'une liberté sans précédent, l'homme n'est pas toujours heureux. Il éprouve un sentiment d'impuissance, car l'autonomie existe de jure (l'homme est libre) mais pas nécessairement de facto (il ne peut rien influencer). En plus, il souffre de la solitude et de l'indifférence, puisque chacun prend soin de lui-même. Dans la société individualisée, les croyances au progrès et à la perfection sont mortes. Le seul perfectionnement possible passe par la réalisation de soi. Comme l'insatisfaction fait partie intégrante de la vie quotidienne et que chacun est responsable de ses propres actes, la critique de la société cède le pas au constant mécontentement de soi-même. L'individu ainsi angoissé se noie dans l'autocritique et l'égoïsme. (BAUMAN, $2000:$ 16-52)

La disparition de l'autorité suprême dote l'homme d'une liberté individuelle tout en le plongeant dans le doute, l'incertitude et l'angoisse. Le monde liquide ressemble à une collection illimitée de possibilités parmi lesquels il faut sans cesse choisir: le tourment ne découle plus de l'absence de possibilités mais de leur profusion. Une seule autorité restrictive a été remplacée par la surabondance d'autorités qui tentent et séduisent l'homme contraint à choisir. En fait, la majorité n'est plus dominée par la minorité, la majorité épie - et copie - la minorité par 
l'intermédiaire des médias. La vie qu'on regarde à la télé paraît être plus réelle que la réalité elle-même et, de ce fait, éclipse la vie quotidienne. La distance efface tous les détails qui s'inscrivent mal dans la Gestalt. On a tendance à voir dans la vie des autres une œuvre d'art et, surtout, à transformer notre propre vie, notre propre identité, en une telle œuvre d'art. Mais « chaque fois que nous parlons d'identité, il y a au fond de notre esprit une faible image d'harmonie, de logique, de cohérence » (BAUMAN, $2000: 82$ ). Comme on manque de toutes ces choses, la quête identitaire est aujourd'hui une peine perdue : il est impossible de solidifier le fluide, de donner forme à l'informe. Et si les possibilités illimitées permettent à l'homme de devenir n'importe qui, elles l'empêchent d'être quelqu'un. «Être n'a effectivement aucune valeur, car il est important d' «avoir ». La consommation, qui est devenue d'ailleurs une dépendance, ne doit plus satisfaire les besoins, elle doit constamment assouvir de nouveaux désirs, instables et de ce fait insatiables. D'où aussi notre propension à l'hédonisme. (BAUMAN, $2000: 53-90$ )

La métamorphose de la relation entre le temps et l'espace représente le signe distinctif de la modernité qui, effectivement, commence au moment où le temps se dilate - grâce à l'invention de l'automobile, tandis que l'espace reste inflexible. À l'époque liquide, l'accélération atteint les limites du possible : le temps est réduit à l'instantanéité. Vu que ce sont la circulation, le recyclage et la substitution qui apportent un revenu, il devient inutile de rester attaché au terrain et aux biens immobiliers : il est facile de quitter ce qu'on peut acquérir sans peine. Se substituant à la durée, l'instantanéité annule l'espace et liquéfie tout corps solide. Chaque instant est infiniment spacieux, puisqu'il n'y a pas de limites à ce qui pourrait être tiré de n'importe quel moment, aussi fugace soit-il. L'homme ne désire plus atteindre l'immortalité à travers ses œuvres, il vit selon la philosophie carpe diem, il est obsédé par son corps et sa satisfaction. En effet, il s'agit des seules entités dont la durée de vie tend à se prolonger. Tout éphémère qu'il est, le corps - et sa satisfaction - est devenu le dernier refuge de la durée. Ainsi, on tient au présent sans néanmoins pouvoir l'influencer d'où la popularité des théories du chaos et des catastrophes. Et moins l'on maîtrise le présent, moins l'on est capable de planifier l'avenir qui ressemble de plus en plus à un labyrinthe : complexe, impénétrable, aléatoire et surprenant. (BAUMAN, $2000: 91-129$ )

Le travail, ce pilier de la modernité solide, perd sa valeur absolue, il n'est plus une vocation ni un axe infaillible autour duquel tourne la vie humaine. À l'ère liquide, le travail devient davantage une distraction, le jeu d'un seul joueur, un acte unique qui doit apporter du plaisir. Le profit n'est plus tiré de la production matérielle mais plutôt spirituelle, le capital ne dépend pas du nombre de travailleurs mais de celui de consommateurs. Contrairement à la production qui nécessite la coopération, la consommation est un acte entièrement individuel. (BAUMAN, 2000 : 130-167)

La disparition des anciennes certitudes entraîne la précarité des relations et des réseaux humains, car des individus incertains sont susceptibles et intolérants. Les relations sont fragiles parce qu'elles ne sont plus produites par le couple, elles sont consommées par l'individu ( « partnerships tend to be viewed and treated as things meant to be consumed, not produced ») : l'objectif en est une jouissance immédiate, dès qu'elle disparaît, l'individu commence une nouvelle relation. L'incertitude 
favorise également la création de communautés privées qui promettent de devenir un port sûr surmontant l'abîme creusé entre la liberté individuelle et l'insécurité. Ces communautés qui réunissent des semblables sont aussi éphémères que les relations individuelles. (BAUMAN, 2000: 168-201) Pierre BOURDIEU le dit clairement : « la précarité est aujourd'hui partout » (1997).

\section{L'existence liquide selon Guillaume Vigneault}

Le monde créé par Guillaume Vigneault dans Carnets de naufrage est aussi liquide que celui décrit par Bauman. Il est peuplé d'individus angoissés et fluides dont notamment Alex, le protagoniste. À vingt-sept ans, il change de partenaire comme de chemise. Arrêter ses études est pour lui aussi facile que s'envoler, du jour au lendemain, au Mexique. Son emploi de barman qu'il exerce trois soirs par semaine ne l'engage à rien ; il s'agit plutôt d'une distraction que d'un travail sérieux. Il aime conduire, jouer au billard, nager et surfer. Il est d'ailleurs un nageur exceptionnel : il a été lancé dans l'eau dès sa naissance (selon une de ces modes new age de l'époque) ce qui a néanmoins fait de lui un être inquiet, caractériel et passablement irascible. Bref, il est un individu liquide.

C'est d'ailleurs aussi le cas de ses amantes, amis et connaissances. Camille, la première amante d'Alex, est une nymphe à l'existence légère: elle voyage beaucoup, étudie l'histoire de l'art et lui parle de «ses idylles légères, ses projets flous » (p. 22). Katarina, son amante serbe, est une «âme féline, fluide, fuyante » (p. 139), à la fois là et ailleurs, « dans une sorte de scaphandre mental » (p. 163), la solitude au fond des yeux.

Une bonne partie des gens qu'Alex rencontre sont soit des ratés, soit ils poursuivent sans conviction une carrière éblouissante et vivent une vie qui n'est pas la leur. Un cas à part est représenté par Yannick qui a hérité d'un restaurant chic à Montréal. À cette époque, il était un gars stressé, toujours en train de se plaindre de tout, dans un constant état de demi-panique, flamboyant et malheureux. Sans le savoir, Alex lui a fait comprendre que tout ce qu'il avait ne valait rien si ça ne le rendait pas heureux. Il a décidé de tout quitter immédiatement et de partir vivre au Mexique. Là, il arrive à élever « la désinvolture au rang d'un art de vivre » (p. 177) et à mener une existence heureuse, même si un peu solitaire.

La fuite est pratiquée aussi par Félix, le seul des amis montréalais d'Alex qui appartient à l'élite de l'ère liquide. Non qu'il soit moins angoissé que les autres personnages, mais il est le seul à profiter du chaos qui règne sur le monde, car son propre modus operandi est la confusion. D'après lui : « La fuite, y a que ça de vrai. » (p. 99) En outre, il remplit les conditions de l'exterritorialité : en mouvement constant, Félix est un dynamiteur. Alex le traite de «circonstancialiste de merde » (p. 144), puisqu'il a, contrairement à lui, une « foi superbe en la flexibilité du réel. » (p. 246) : « Des êtres immobiles, ça n'existe pas. Il n'y a pas d'essence intrinsèque aux gens, aux choses, aux événements ; juste des circonstances, man...» (p. 144). Félix, son nom l'indique, se prête bien à la vie dans le monde liquide.

Le troisième ami important d'Alex est Bernard, le météorologue qu'il rencontre au Mexique et qui lui donne des leçons de surf. Lui aussi a fui sa vie ancienne - sa 
femme et son travail pour la NASA - qu'il a échangée contre le surf, le bateau et une liberté absolue.

C'est avec ces trois amis qu'Alex discute de la nature de l'existence; ce sont eux qui lui montrent comment surmonter sa «détresse innommable» (p. 31) et, surtout, son incapacité viscérale de vivre après le départ de Marlène, sa femme. Toutefois, contrairement à ce qui peut parâtre, son angoisse n'est pas le résultat du naufrage conjugal, elle en est la cause. Les amis d'Alex lui expliquent que sa femme l'a quitté à cause de ses absences, parce qu'il n'était plus là pour elle. Sa perplexité, qui est souvent prise pour de l'indifférence, tire sa source dans le doute, l'incertitude et l'insécurité. Alex est un indécis qui doute « de tout et de son contraire» (p. 153). Il s'est marié pour échapper à la «liberté informe et monstrueuse» (p. 24) qui l'inquiète depuis toujours. Être de nouveau maitre de son existence est pour lui une « une liberté fulgurante, terrible» (p. 39).

Pour éviter la détresse et l'angoisse, Alex opte pour trois stratégies majeures. Il se noie dans l'hédonisme. Il cherche un plaisir éphémère dans la consommation démesurée d'alcool, de nourriture, d'amour, d'amitié, de vitesse, de jeu, d'effort. Mais ses excès ne le tirent pas de sa solitude et l'enfoncent encore plus profondément dans le désespoir et la dépression. Toutes ses tentatives de suicide par noyade sont également des actes manqués. Comme il répète cette épreuve par pur «plaisir masochiste» (p. 178), il n'arrive pas à remplir le vide béant au fond de lui. La fuite, tellement appréciée par ses amis Félix, Yannick et Bernard, semble être une bonne option. En effet, lors de son séjour au Mexique, Alex apprend comment cueillir le jour :

« Je me serais fait à cette existence [...]. Ne rien vouloir d'autre que ce que je pouvais vivre dans une journée ; m'appliquer, me faire un devoir de ne rien désirer au-delà d'un bonheur simple, un bonheur à hauteur d'homme. Cela me semblait être un noble projet. Fuir. J'ai songé qu'une belle fuite ça valait quelque chose. » (p. 221)

Pourtant, il finit par comprendre que sa vie est à Montréal et qu'une nouvelle vie ailleurs, qui serait nécessairement fausse, le tuerait à petit feu. Son retour à Montréal et sa rencontre finale avec Marlène, de nouveau disponible, pourraient donner l'impression que le héros n'a pas changé : il est toujours aussi indécis. Les phrases finales du livre en témoignent : «Je ne sais pas, Marlène, [...]. Je ne sais pas. » (p. 264) Et pourtant, les mêmes mots révèlent le contraire. Tout au long du livre, Alex souffre de sa rupture, pense à Marlène tout le temps, désire sa proximité. Le fait qu'il ne se jette pas dans ses bras pour remplir le vide, pour mettre fin à sa liberté, prouve qu'il a évolué. Toutes ses expériences lui ont finalement appris quelque chose. Le surf y est pour beaucoup. Il comprend qu' « une manifestation du chaos ne [peut] être prévue que par une perception également chaotique » (p. 165). On ne peut comprendre la mer qu' « avec le ventre » (p. 233) : le calcul n'y est pour rien, il faut de l'instinct, laisser vibrer ses sens librement. En surfant, Alex apprend à utiliser son corps « comme un médium » l'aidant à « décrypter ainsi les couches brutes de l'existence, sans mots. » (p. 233). Il ne lui reste qu' à appliquer ce nouveau savoir-faire à la vie. Félix le lui dit d'ailleurs assez clairement : «Vis-le et ferme ta gueule» (p. 165). 


\section{La métaphore liquide}

Pour capter l'existence liquide, Guillaume Vigneault crée un réseau métaphorique dense et complexe grâce auquel l'eau submerge l'œuvre entière, dans sa microstructure aussi bien que sa macrostructure. Le fait que l'eau constitue le noyau des champs lexicaux n'est pas surprenant: la moitié du roman se passe au bord de la mer. Mais l'eau est omniprésente aussi dans le reste du roman qui se passe à Montréal dont le caractère insulaire est pourtant passé sous silence. En fait, l'eau - ou son absence - noie les métaphores figées, les métaphores filées ainsi que les images, ces passages hautement poétiques où Alex soit entre en contact direct avec l'élément liquide (sous forme de pluie ou de mer), soit rêve du désert. Et finalement, par son mouvement, l'eau structure la trame narrative.

Assez logiquement, les métaphores figées, qui font le plus souvent référence à l'eau, apparaissent dans le contexte de la souffrance : le roman entier se déroule sous le signe de la souffrance. Ce qui frappe, par contre, c'est le mouvement - ou son absence - découlant du choix des formulations concrètes. Ainsi, les expressions peuvent être réparties en deux groupes dont le premier implique la mobilité, tandis que le second, beaucoup moins fréquent, l'immobilité. L'idée de la mobilité, elle, est très peu exprimée par des locutions connotant un mouvement relativement stable et continu comme quand le héros baigne dans un douillet coma pendant un mois ou quand son ex-femme, Marlène, nage dans l'ostensible euphorie des débuts d'une nouvelle relation. Beaucoup plus fréquentes sont les expressions qui actualisent l'idée du changement de direction ou d'état. Du couple antithétique apparition/disparition, le premier élément est pratiquement ignoré, alors que le second afflue. Alex s'engouffre ou se sent plongé quelque part, Katarina est immergée en elle-même et Marlène saborde «le navire plutôt que de juste couler avec» (p. 79). Encore plus souvent, le héros se sent aspiré. Sa souffrance est « un gouffre béant» (p. 16) qui s'ouvre devant lui, sous lui, en lui. Camille est un vortex qui aspire son regard, Marlène a des instants comme des gouffres auxquels il doit s'accoutumer. De plus, Alex a de la peine à avaler et à absorber ce qui lui arrive. L'actualisation du potentiel sémantique liquide passe aussi par les renvois au changement d'état: l'ivresse, la révolte, le désert et la phrase s'évaporent, les conversations avec Marlène se diluent dans de longs silences et tout se liquéfie au moment où Alex apprend la rupture de Marlène avec Jean.

Si l'eau et la liquidité véhiculent la mobilité et le changement, le manque d'eau connote généralement l'immobilité et l'état. En décrivant sa gueule de bois, Alex parle du «Sahel de [son] métabolisme » (p. 62). Les conversations avec Camille et, surtout, avec Marlène sont sèches. Marlène est d'ailleurs souvent associée à l'image du désert. Leur lit conjugal est un désert. Quand il pense à elle, Alex constate qu'il y a « un désert en [lui], infini, immobile, une peine tranquille » (p. 210), tandis qu'en écoutant Camille, la vie redevient « un vaste pré » (p. 22).

Les métaphores filées, elles, établissent un lien solide entre l'eau et la vie. La vie est comparée au gouffre :

« J'ai vu un gouffre. Ma gorge s'est resserrée. Et pourtant, ce n'était rien. C'était la vie, qui se déroulait bêtement, sans surprise. La vie, celle qui nettoie derrière soi les miettes de pain qu'on a laissées tomber. Le vent et les traces du loup dans la neige. Ce n'était rien. » (p. 125-6) 
La relation d'Alex avec Camille est une navigation qui tire à sa fin. Il a l'impression de s'être embarqué clandestinement mais tant qu'on voudrait bien lui « foutre la paix question passeport, billet, [son] rafiot tiendrait la mer. Ça grondait au large, mais pour l'instant c'était une mer d'huile. Chaude. » (p. 32). Lors de la relation sexuelle avec Katarina, tout l'aspire, le submerge, le noie. Un «gouffre sulfureux » (p. 151) se forme entre leurs corps et l'entraîne mille mètres sous l'eau. Il est « serein alors que [ses] poumons implosent, seul aussi. » (p. 151) Le départ de Marlène déclenche chez lui une vision de la vie à quarante-cinq mille pieds où la température moyenne est de moins cinquante-trois degrés. Là-haut, les nuages sont « composés de minuscules cristaux de glace en suspension ». Et il pense «qu'on devait se sentir très petit, là-haut ; très petit, oui, et très seul aussi. » (p. 84) La discothèque est apparentée à une "marée humaine », à un "aquarium géant » où l'air est tellement saturé qu'il en devient «presque liquide» (p. 223). C'est d'ailleurs le monde entier qui ressemble à un aquarium de piranhas où les hommes, tels les poissons rouges et tel Sisyphe, sont condamnés à nager "aux côtés du bourreau, continuant à vivre, à bouffer, à baiser malgré la mort imminente. » (p. 253) L'eau des métaphores filées aspire, engloutit et saborde, elle connote la solitude, l'éphémère et, surtout, la mort.

L'eau qui donne la vie aussi bien que la mort s'inscrit dans le roman dès les premières pages : la voiture d'Alex est arrêtée par « un de ces orages apocalyptiques [sic !] de juillet » (p. 11) et garée « au milieu de nulle part, sur une mince bande de bitume, entre deux champs de maïs » (p. 11) aux allures d'océan. Alex essaie de redémarrer mais n'insiste pas. Il descend de la voiture et se laisse imprégner par l'eau de la pluie « tiède, presque chaude » (p. 12), il attend la foudre tout en sachant que ses chances d'en être touché sont infinitésimales, décevantes. Une fois l'orage passé, Alex arrive à redémarrer, après plusieurs essais, le moteur toussotant. Dans cette image initiale, le voyage d'Alex est implicitement mis en parallèle avec son existence et anticipe le « cours tortueux de sa vie » (p. 34). Avec chaque réapparition de la crise, Alex se laisse imprégner par l'eau tout en attendant la mort qui ne vient pourtant pas. Les images suivantes en témoignent : sa nage dans la houle glaciale dans le Maine (chapitre 7), sa nage dans le ressac au Mexique (chap. 26), ses leçons de surf dans des remous et des vagues vertigineuses (chap. 33 et 36) toujours au Mexique ne sont que des tentatives de suicide manquées. Incapable de vivre, Alex est également incapable de mourir.

Si l'existence d'Alex est successivement rapprochée du voyage, de la nage et du surf, la vie est métaphorisée tantôt en mer, tantôt en désert, tantôt les deux. Cela est en partie lié au fait que les images liquides alternent avec les séquences oniriques (chap. 10, 27, 41) où Alex marche dans le désert et gravit les dunes. Mais il n'est pas rare que la mer prenne les allures d'un désert et inversement, cela dans les deux types d'images. Ainsi, la houle fait "penser à des dunes liquides et mouvantes » (p. 44), tandis que les «dunes ondulent mollement» (p. 60). Dans l'image initiale (Incipit), la mer est associée à la vie actuelle d'Alex, alors que le désert à sa vie conjugale qui n'est plus la sienne. Dans son premier rêve de désert (chap. 10), Alex fait « semblant de ne pas voir le lent mouvement de la houle » : « si j'accepte l'idée que les dunes bougent, le sable va m'avaler » (p. 60). De fait, Alex refuse d'accepter 
la nature liquide de l'existence bien qu'il en soit conscient. Mais après sa dernière leçon de surf (chap. 36) où il dompte, ou plutôt apprivoise la mer, il se rend compte que le désert n'a rien à voir avec Marlène : il est en lui. Cela est scellé dans le troisième rêve de désert (chap. 41) par la disparition symbolique de la femme, par sa solitude prononcée et surtout par la transformation du désert en océan :

« Je gravis une pente, je m'enfonce jusqu'aux genoux dans le sable brûlant. Jusqu'à la taille. Jusqu' au cou. Je nage à présent, la planche flotte sur le sable, je m'y agrippe. [...] Une dune passe sous moi, une onde me soulève. [...] Cette vague m'appartient, je la possède [...]. » (p. 230)

S'il regarde en arrière, tout s'arrête, immobile. Quand il accepte le mouvement des dunes, il se laisse avaler par elles et « tout devient clair » (p. 230). Il est en plein océan, ne voit pas la côte et se demande si « elle existe encore, cette côte, si tout n'est plus qu'océan. » (p. 230). Il finit par comprendre que le monde est liquide, qu'il l'a été même avant son naufrage et qu'il vaut mieux se l'approprier.

Il est à noter que la métaphorisation de la vie est étroitement liée à la mobilité. Si l'incipit s'ouvre par un arrêt symbolique, les autres images actualisent l'idée d'un mouvement chaotique, qui passe par toutes les directions - la nage dans la houle glaciale finit par une dérive (chap. 7), la marche dans le désert dont rêve Alex implique le mouvement soit en avant (chap. 10), soit vers le haut (chap. 27), la nage dans le ressac entraîne ce que les surfeurs appellent la lessiveuse (chap. 26). Ce mouvement en spirale se répète d'ailleurs avec les deux leçons de surf (chap. 33 et 36). Alex se laisse tirailler sans combattre par les « courants rageurs » (p. 158). « Meurtri, battu, exténué à [s]'en vomir les poumons » (p. 189), il s'obstine et finalement arrive à chevaucher les vagues vertigineuses. Le dernier rêve de désert (chap. 41) intègre la mobilité chaotique des images précédentes: quand Alex regarde en arrière, tout s'arrête, ensuite il s'enfonce dans le sable devenu océan, il y nage, il se lève sur sa planche et chevauche un tsunami de cent mètres. Cette vague, qui lui appartient, le transporte, docile et bienveillante, à mille kilomètres-heure.

La mobilité trouve son écho également dans la structuration de la trame narrative. En effet, l'ancrage spatial des chapitres reproduit le mouvement, répétitif mais irrégulier, de l'océan. Le récit s'ouvre par l'arrivée à Montréal (Incipit sans numérotation) où Alex passe un certain temps (chap. 1-5). Ensuite, il voyage avec Camille dans le Maine où ils restent une dizaine de jours (chap. 6-10). Le retour à Montréal met fin à leur relation (chap. 11). Alex passe l'automne à Montréal (chap. 12-25). Après une hibernation, il part pour le Mexique avec Katarina. Elle rentre après quelques jours mais il y demeure trois semaines (chap. 26-43). Finalement, il retourne à Montréal (chap. 44-46) où il s'installe de nouveau (chap. 47-50).

Le premier retour d'Alex à Montréal marque le changement de sa perception de la ville qui lui paraît «neuve, factice même. Une brillante contrefaçon de celle qu' [il connaissait] » (p. 14). À son dernier retour, Alex constate par contre que rien n'a changé depuis son départ : «Mêmes visages, même décor, mêmes discussions stériles » (p. 248). Il a l'impression «d'avoir vécu trois ans ailleurs », pendant que tout le monde à Montréal a «tout juste eu le temps de boire une bière. » (p. 248) Cette fois, ce n'est pas la ville ni sa perception qui changent, c'est Alex. La 
quatrième de couverture du roman l'explique bien: Alex a été à la dérive, une «douce dérive». Emporté par un courant inférieur, Alex finit par s'ouvrir à l'existence liquide.

\section{Entre deux eaux}

Maints aspects relient Carnets de naufrage aux romans de Toussaint et de Houellebecq. Pareillement à La salle de bain, l'eau omniprésente imprègne le lexique, les figures de style, la trame narrative ainsi que les réflexions philosophiques. Néanmoins, le récit de Toussaint est linéaire ( $c f$. la numérotation des paragraphes), tandis que celui de Vigneault non-linéaire : non seulement les déplacements dans l'espace véhiculés par les images vont dans tous les sens, nous y trouvons plusieurs références au chaos y compris le bar appelé le Chaos Theory. Chez Toussaint, la linéarité est enrichie par le mouvement cyclique ( $c f$. la double chronologie du récit étudiée par DELANNOI, 1985 dans DEMOULIN, 2005 : 13), sa structure tripartite (Paris - Venise - Paris) véhicule l'idée de l'éternel retour. Chez Vigneault, par contre, la structure actualise l'idée de la dérive, même si symbolique puisqu'intérieure : le point de départ et d'arrivée est le même, mais le protagoniste est différent. Ce qui caractérise le héros de Vigneault, c'est sa mobilité qui contraste vivement avec l'immobilité du héros de Toussaint. ${ }^{2}$ Et si La salle de bain raisonne sur la fuite de temps et la durée, les Carnets de naufrage reflètent l'instantanéité.

En vérité, le narrateur des Carnets de naufrage tient davantage du narrateur de l'Extension du domaine de la lutte. Les deux sont plongés dans l'eau froide et amère, sauf que les noyades d'Alex sont des actes délibérés. Loin du bord, le narrateur houellebecquien continue à nager même s'il ne croit plus à l'existence d'une autre rive et que sa vie ressemble de plus en plus à une noyade. Alex, par contre, se remet à nager précisément au moment où il cesse de se poser la question de l'existence d'une autre rive. Chez Houellebecq, c'est le narrateur qui déclenche le mécanisme du souvenir en se plongeant symboliquement avec son lecteur dans l'eau froide. Chez Vigneault, le plongeon est symbolique aussi bien que littéral et c'est l'eau glaciale qui déclenche le mécanisme du souvenir semblable à celui de la madeleine proustienne, seulement l'objet mouillé de thé cède le pas au sujet enfoncé dans l'eau et l'agréable cède le pas au désagréable.

Le monde dans lequel vit le héros des Carnets de naufrage est une copie conforme de celui décrit par Zygmunt Bauman. Dans son roman, Vigneault a bien saisi les cinq piliers sur lesquels, d'après Bauman, repose la différence entre la société solide et liquide. L'émancipation de l'homme est symboliquement exprimée par le départ de Marlène. Le héros, ainsi libéré de la/sa société, est sujet à l'angoisse, la solitude et l'indifférence qu'il essaie de combattre par un égoïsme marqué. Le départ de Marlène prive aussi le héros de l'autorité «suprême » ce qui le laisse en proie au doute et à l'incertitude. Il cherche les conseils chez d'autres autorités,

\footnotetext{
${ }^{2}$ Il est à noter que le modèle du protagoniste toussaintien trouve son écho plutôt dans le deuxième roman de Guillaume Vigneault, Chercher le vent (2001) : son héros, qui est comme Alex un individu parfaitement liquide, a une préférence marquée pour les espaces clos et, malgré ses voyages, arrive à rester immobile.
} 
représentées par ses amis Félix, Yannick et Bernard. Il regarde comment ils vivent et les imite : il pratique la fuite à la manière de Félix, le carpe diem à la Yannick, l'intuition à la Bernard. Il se trouve capable de devenir comme eux sans être capable de véritablement être tel qu'eux. C'est pourquoi il dit «Je me serais fait à cette existence » (p. 221) quand il parle de sa vie au Mexique. Après le départ de Marlène, il vit dans l'instantanéité, il n'est attaché à rien et il est obsédé par son corps et sa satisfaction. Son hédonisme répond à son besoin d'assouvir constamment de nouveaux désirs. Son travail et tout ce qu'il fait relève de la distraction et du jeu. Après le départ de Marlène, ses relations sont d'une précarité très prononcée. Il ne veut pas participer à leur construction, il préfère les « consommer » en solitude. Dès que disparaît la jouissance, il y met fin, déjà en quête d'un nouvel assouvissement de ses désirs.

Marlène est pour Alex un point de repère. Sa vie avec elle est dès le début associée à la solidité. Marlène est le désert, immobile et immuable comme le marbre. Après le départ de Marlène, la vie d'Alex est, tout comme sa voiture, garée « au milieu de nulle part, sur une mince bande de bitume, entre deux champs de maïs » (p. 11), entre deux existences, entre deux mondes : le premier, solide et immobile, n'est plus le sien et le second, liquide et en mouvement perpétuel, n'est pas encore le sien. La métaphore de l'entre-deux réapparaît deux fois : lors de la nage dans l'eau glaciale quand Alex se souvient de sa rencontre avec la mort, incarnée par le requin, et lors de la sortie de surf quand une vague l'enferme dans son intérieur. Dans ces deux cas, Alex est «suspendu entre deux eaux » (p. 41). En outre, les références intertextuelles vont dans le même sens. Alex lit Paradis perdu d'Hemingway et écoute Break on through des Doors. D'une part, une œuvre classique dont le titre est bien significatif ; d'autre part, une chanson moderne au titre tout aussi sémantiquement chargé, en plus caractérisée comme « sorte de bossanova apocalyptique » (p. 222). Rappelons que bossa-nova signifie en portugais du Brésil « nouvelle vague » (LAROUSSE).

Or, ces deux champs de maïs entre lesquels est coincée l'existence d'Alex ondoient sauvagement et ressemblent à l'océan : dès l'incipit, le solide est apparenté au liquide. Ce lien est ensuite explicité dans les passages introspectifs. Ainsi, le premier rêve de désert révèle qu'Alex est conscient de la nature liquide du monde et de la vie, avec ou sans Marlène, mais il a encore peur de l'accepter. Dans le dernier rêve, qui est davantage une vision, le désert se transforme graduellement en océan. En effet, Alex a fini par comprendre que le désert, infini et immobile, est en lui et que le monde est liquide : il faut s'y adapter, accorder son rythme à la houle, chevaucher les vagues. Bref, il faut s'approprier le monde mouvant. 


\section{BIBLIOGRAPHIE}

BAuman Zygmunt (2000), Liquid Modernity, Cambridge, Polity.

BOURDIEU Pierre (1997), «La précarité est aujourd'hui partout », Intervention lors des Rencontres européennes contre la précarité, décembre 1997 [En ligne]. http://www.ilo.org/wcmsp5/groups/public/@ed_dialogue/@actrav/documents/ meetingdocument/wcms_161352.pdf [consulté le 5 novembre 2019].

DelanNOI Gil (1985), «Cruel Zénon », Critique, no 463, décembre 1985, cité dans Laurent DEMOUlin, La salle de bain, revue de presse établie par Laurent Demoulin [En ligne]. Paris, Minuit, 2005. http://www.jptoussaint.com/documents/c/cd/051186_i_salle_bain.pdf [consulté le 14 novembre 2019].

JUIGNET Patrick (2017), «La modernité liquide de Zygmunt Bauman», Philosophie, science et société, 4 février 2017 [En ligne]. https://philosciences.com/234-la-modernite-liquide-de-zygmunt-bauman [consulté le 31 octobre 2019].

LAROUSSE, « Définitions : bossa-nova, bossas-novas - Dictionnaire de français Larousse »[En ligne]. https://www.larousse.fr/dictionnaires/francais/bossanova/10301?q=bossa+nova\#10176 [consulté le 14 novembre 2019]. 